\title{
Asymptomatic domestic dogs are carriers of Leishmania infantum: possible reservoirs host for human visceral leishmaniasis in southern Iran
}

\author{
Mahdi Fakhar • Mohammad Hossein Motazedian • \\ Qasem Asgari • Mohsen Kalantari
}

Received: 9 April 2010 / Accepted: 8 February 2011 /Published online: 22 February 2011

(C) Springer-Verlag London Limited 2011

\begin{abstract}
In the past few years, the incidence of human visceral leishmaniasis (HVL) has increased in many districts of Fars Province, southwest of Iran, particularly, among communities of nomadic tribes. Recent epidemiological reports in Leishmania infantum endemic regions of Iran indicate that more than $50-70 \%$ of seropositive dogs are asymptomatic for Leishmania infection. Between 2004 and 2006, blood samples were collected from 110 domestic dogs from nomadic and rural areas. Each of these samples was tested for anti-Leishmania antibodies, in direct agglutination tests (DATs), and for L. infantum kinetoplast deoxyribonucleic acid (kDNA), in polymerase chain reaction (PCR)-based assays. Of the 110 dogs, 5.5\% (6/110) were found seropositive and 23\% (25/110) PCR-positive. Four of the six seropositive (67\%) and 22 of the 25 PCRpositive ( $88 \%$ ) were asymptomatic. The rate of infection in dogs from nomadic communities was higher $(27 \%)$ than dogs from rural areas (18\%). Since positivity in the PCRbased assay indicated the presence of $L$. infantum amastigotes in the peripheral blood of $23 \%$ of the subjects, it is clear that these asymptomatic dogs (88\%) are quite
\end{abstract}

\section{Fakhar}

Department of Parasitology and Mycology, School of Medicine, Mazandaran University of Medical Sciences,

Km 18, Khazar-Abad Road, P.O. Box 48175-1665, Sari, Iran

\section{Fakhar $(\bowtie)$}

Molecular and Cellular Biology Research Center,

Mazandaran University of Medical Sciences,

Km 18, Khazar-Abad Road, P.O. Box 48175-1665, Sari, Iran

e-mail: mahdif53@yahoo.com

M. H. Motazedian · Q. Asgari $\cdot$ M. Kalantari

Department of Parasitology and Mycology, School of Medicine,

Shiraz University of Medical Sciences,

Shiraz, Iran common in the study areas and probably act as reservoirs in the transmission of Leishmania parasites, to humans and to other dogs, by sandflies. Moreover, our study showed that application of PCR to buffy coat samples gave a better estimate of the real rate of infection in asymptomatic dogs than DAT.

Keywords Canine visceral leishmaniasis · Asymptomatic dogs $\cdot$ Leishmania infantum, Polymerase chain reaction . Direct agglutination test

\section{Introduction}

Visceral leishmaniasis (VL) or kala-azar is a systemic disease which is caused by Leishmania donovani complex. It is estimated that the annual incidence of human visceral leishmaniasis (HVL) cases worldwide is about 500,000 cases (World Health Organization 1998).

Visceral leishmaniasis in the countries of the Mediterranean basin and the Middle East including Iran is caused by Leishmania infantum (Mohebali et al. 2005). In Iran, the main endemic foci for VL are Fars and Bushehr Provinces, in the southwest, the districts of Meshkin-shahr and Kaleybar in the northwest and Qom Province in the center of Iran (Asgari et al. 2006; Mohebali et al. 2001, 2005; Fakhar et al. 2004, 2006, 2008).

Commonly, domestic dogs (Canis familiaris) are the most important domestic reservoir hosts for Mediterranean visceral leishmaniasis (MVL; Mazloumi Gavgani et al. 2002). L. infantum infection in dogs ranged from $10 \%$ to $37 \%$ in the Mediterranean basin (Fisa et al. 1999; Sideris et al. 1999; Mohebali et al. 2005). In Iran, canine visceral leishmaniasis (CVL) is endemic in northwest, southwest, and central parts, and the incidence varies between $4.5 \%$ 
and 18\% (Edrissian et al. 1996; Mohebali et al. 2001, 2005; Fakhar et al. 2004, 2006).

The majority of seropositive dogs are asymptomatic, but others have cachexia, weight loss, enlargement of lymph nodes, splenomegaly, skin and eye lesions, alopecia, renal failure, hemorrhagic diarrhea, nose bleeding (epistaxis), and abnormal nails (onychogryposis; Koutinas et al. 1999). Recent epidemiological reports in endemic regions of CVL, such as Iran, indicate that asymptomatic dog infections with L. infantum occur in more than $50-70 \%$ of the seropositive dogs in the field investigations (Mohebali et al. 2005; Fakhar et al. 2006; Moshfe et al. 2008).

The Canidae families are main reservoir hosts because the parasites multiply in macrophages in the skin and are readily transferred by feeding sandflies (Ashford et al. 1998). In addition, the wild carnivores, such as jackals and foxes, are considered reservoir hosts in sylvatic cycle of MVL, principally in sporadic areas of Iran (Mohebali et al. 2005).

Specific diagnostic techniques for canine leishmaniasis include microscopy, culture, serology, polymerase chain reaction (PCR), and xenodiagnosis. Canine leishmaniasis in infected dogs may be asymptomatic and seronegative (Molina et al. 1999), so detection of parasites by a molecular method, such as PCR, may be useful to detection of asymptomatic infections. In the past few years, there were many reports of new HVL cases from most parts of Fars Province that suggest that the incidence of HVL is increasing in these areas especially among communities of nomadic tribes. Dogs are very important in the life of these communities and there is a close human-dog association. The mean seroprevalence of HVL in Fars Province is 1.6\%. However, there is no data on prevalence of Leishmania infection in dogs, as a main reservoir hosts, especially among nomadic tribes. The aim of the present study was to investigate the prevalence of asymptomatic and symptomatic domestic dogs (including rural and nomadic population) infected with $L$. infantum in the province by using direct agglutination tests (DATs) and PCR-based assays to test blood samples from all infected subjects for the evidence of recent or current infection.

\section{Subjects and methods}

\section{Study area}

Fars Province is located in the southwest of Iran and has an area of about 53,000 miles ( $8 \%$ of Iran's area) and is located at $27^{\circ} 3^{\prime} 42^{\prime \prime} \mathrm{E}$ longitude. It is mountainous with an average elevation of 5,000 feet above sea level. The climate is quite dusty and dry, with warm summers, generally mild winters, and a great deal of sunshine throughout the year. Three important nomadic tribes including Qashqai, Arab, and Lur live in this province. Nomadic tribes keep dogs which are important for herding their animals. These tribes migrate regularly between summering and wintering quarters. As they move about $300 \mathrm{~km}$ from the southeast to the east part (Fars Budget and Planning Organization 2003) and back, they could play a significant role in spreading the VL infection.

\section{Blood sampling}

Blood samples were collected from 110 domestic dogs (61 females and 49 males) from rural and nomadic areas in Fars Province, as an endemic area of HVL, throughout 2004 2006. Blood ( $2 \mathrm{ml})$ were collected in ethylenediaminetetraacetic acid (EDTA)-coated tubes. The blood was centrifuged at $1,000 \times \mathrm{g}$ for $5 \mathrm{~min}$, and plasma and buffy coat (BC) were separated and stored at $-20^{\circ} \mathrm{C}$. Dog age was determined by interviewing dog owners. Male dogs constituted $69.7 \%$ of the sampled population and females $30.3 \%$. No transmission of Trypanosama cruzi was reported in dogs in the studied areas.

\section{Serological test}

The plasma samples from dogs were tested by DATs as described by Harith et al. (1989). The plasma was diluted 1:160 for preliminary screening and the positive samples were serially diluted up to $1: 1,240$ to obtain the real titer for IgG antibody titration. The cutoff of DAT for CVL was considered 1:320 titers. The highest titers at which agglutination was still visible was reported.

Parasitological study

Nine of the suspected CVL-positive dogs were dissected after obtaining owner consent. Impression smears were provided from the spleen, liver, and bone marrow of these dogs and then stained with Giemsa and examined under a compound light microscope for the amastigote forms (Leishman bodies). Samples from these tissues were also inoculated into the Evans medium and incubated at $25^{\circ} \mathrm{C}$ for 1-4 weeks with weekly subcultures (Evans 1989). Samples were examined regularly to monitor the growth and presence of contaminations.

\section{DNA extraction}

Total DNA was extracted from blood BC as described by Motazedian et al. (2002). Briefly, $200 \mu \mathrm{l}$ of BC was homogenized with $200 \mu$ lyses buffer [50 mM Tris- $\mathrm{HCl}$ (pH 7.6), $1 \mathrm{mM}$ EDTA, and 1\% Tween 20] and $10 \mu \mathrm{l}$ of proteinase K solution (containing $19 \mathrm{mg}$ of the enzyme $/ \mathrm{ml}$ ), in a $1.5 \mathrm{ml}$ microcentrifuge tube. The homogenate was then 
incubated at $37^{\circ} \mathrm{C}$ overnight before $200 \mu \mathrm{l}$ of a phenol: chloroform:isoamyl alcohol mixture was added. After being shaken vigorously, the tube holding the mix was centrifuged $(10,000 \times g$ for $10 \mathrm{~min})$, and then the DNA in the supernatant solution was precipitated with $400 \mu \mathrm{l}$ cold, pure ethanol, resuspended in $50 \mu \mathrm{l}$ double-distilled water and stored at $4^{\circ} \mathrm{C}$ until it could be tested.

\section{PCR assay}

To detect dogs' infection rate for Leishmania spp., the PCR protocol that was previously described by Lachaud et al. (2001) was used. In this step, primers RV1 (5CTTTTCTGGTCCCGCGGGTAGG-3) and RV2 (5CCACCTGGCCTATTTTACACCA-3) were used to amplify a fragment of $145 \mathrm{bp}$ present on the highly multicopy kinetoplast deoxyribonucleic acid (kDNA) minicircles.

The set of primers LIN-4 (forward: 5'-GGGGTTGG TGTAAAATAGGG-3'), LIN-17 (reverse: 5'TTTGAACGGGATTTCTG-3') was used for speciesspecific PCR to characterization of Leishmania species. The same protocol that was described by Azizi et al. (2006) was used in this study. The results were analyzed by SPSS software using Chi-square test and a $P$ value $<0.05$ was considered statistically significant.

\section{Results}

Serological and parasitological screening

Using the DAT, the seroprevalence of specific antiLeishmania antibodies was $5.5 \%$. The seropositivity rate in dogs settled in nomadic and rural areas of Fars Province were equal (Table 1). The rate of the infection among male and female dogs was $6.5 \%$ and $4.1 \%$, respectively, but no statistically significant difference between them was observed $(P=0.35)$. The highest prevalence was observed in 1 - to 5 -year-old dog age group $(6.4 \%)$, but this was not statistically significant. Only $8.1 \%(9 / 110)$ had clinical signs. All seropositive cases with a titer $\geq 1 / 320$ belonged to nomads. Clinical signs including alopecia, cachexia, skin lesion, and weight loss were observed in two of the six seropositive dogs (see Table 2).

Amastigotes (Leishman-Donovan bodies) of Leishmania sp. were observed in the impression smears of the spleen, liver, and bone marrow of two out of nine dogs that were killed, both of which were seropositive. Only one isolate out of samples from two dogs showed exponential growth on Evans medium.

\section{PCR screening}

A number of 11/43 (26\%) of dogs belonging to nomads and $14 / 67$ (21\%) of dogs from rural areas were PCR-positive with the specific primers RV1 and RV2 (Fig. 1). The global prevalence was $23 \%$ and the difference in prevalence between dogs from nomadic groups and rural areas was not significant $(P=0.38$; Table 1$)$.

The highest prevalence $(66.7 \%)$ was observed in dogs over 5 years old, but this was not statistically significant. However, the rate of infection in $<1$-year-old dogs $(46.1 \%$, $6 / 13)$ and 1 - to 5-year-old dogs $(18 \%, 17 / 94)$ had statistically significant differences with others $(P=0.03$ and $P=0.04$, respectively). Only $12 \%(3 / 25)$ of the PCRpositive had clinical signs. No statistically significant differences were observed between PCR-positive dogs and clinical signs ( $P=0.67$; Table 2).

Using Evans medium for culture, the organism was isolated from an animal and then PCR method was performed to characterize the parasites, as described above. Using species-specific PCR with LIN-4 and LIN-17 primers on $\mathrm{BC}$ and also isolated Leishmania parasite, confirmed the parasites as L. infantum (Fig. 2).

\section{Discussion}

Our results showed 5.5\% seroprevalence rate and a PCRpositive rate of which $23 \%$ is markedly higher than that detected by DAT. Since DAT was described as a simple and suitable serodiagnostic test for large-scale screening of CVL in dog populations (Harith et al. 1989; Edrissian et al. 1996), it was used in the present survey.

PCR has high sensitivity and specificity $(100 \%)$ for large-scale epidemiological field studies (Moreira et al. 2007), so it was used in this survey to detect asymptomatic and symptomatic canine leishmaniasis. It is also useful to complement the serological results (Maia et al. 2009). Only a weakly positive correlation was found between the seropositivity and PCR-positive (3 out of 28). These finding were the same as other studies. In Greece, 22 out
Table 1 The numbers of CVL cases from Fars Province found seropositive in the DAT and PCR-positive for the kinetoplast DNA of Leishmania infantum

\begin{tabular}{llcc}
\hline Area & No. of subjects & PCR-positive & DAT titer $\geq 1 / 320$ \\
\hline Nomadic areas & 55 & $15(27 \%)$ & $3(5.5 \%)$ \\
Rural areas & 55 & $10(18 \%)$ & $3(5.5 \%)$ \\
Both & 110 & $25(23 \%)$ & $6(5.5 \%)$ \\
\hline
\end{tabular}


Table 2 The correlation of clinical signs, DAT, and PCR findings in studied dogs from Fars Province

\begin{tabular}{llll}
\hline Clinical signs & No. of subjects & PCR-positive & DAT titer $\geq 1 / 320$ \\
\hline Presented clinical signs & 9 & $3(33 \%)$ & $2(22 \%)$ \\
Alopecia & 6 & 1 & 2 \\
Cachexia & 2 & - & 2 \\
Skin Lesion & 4 & 2 & 2 \\
Weight Loss & 3 & 3 & 2 \\
Lack of clinical signs & 101 & $22(21 \%)$ & $4(3.9 \%)$ \\
Both & 110 & $25(23 \%)$ & $6(5.5 \%)$ \\
\hline
\end{tabular}

of 67 were concordant (Solano-Gallego et al. 2001). In another study, similar to our study, PCR was found positive in 46/73 (63\%), whereas immunofluorescence antibody test only in $9 / 73(12.3 \%)$ of the dogs (Leontides et al. 2002).

In this study, the rate of infection in male dogs was close to that in female dogs by PCR and serology method. Similar results were found by Sideris et al. (1999) in Greece and Mohebali et al. (2005) in Iran. In our study, using PCR

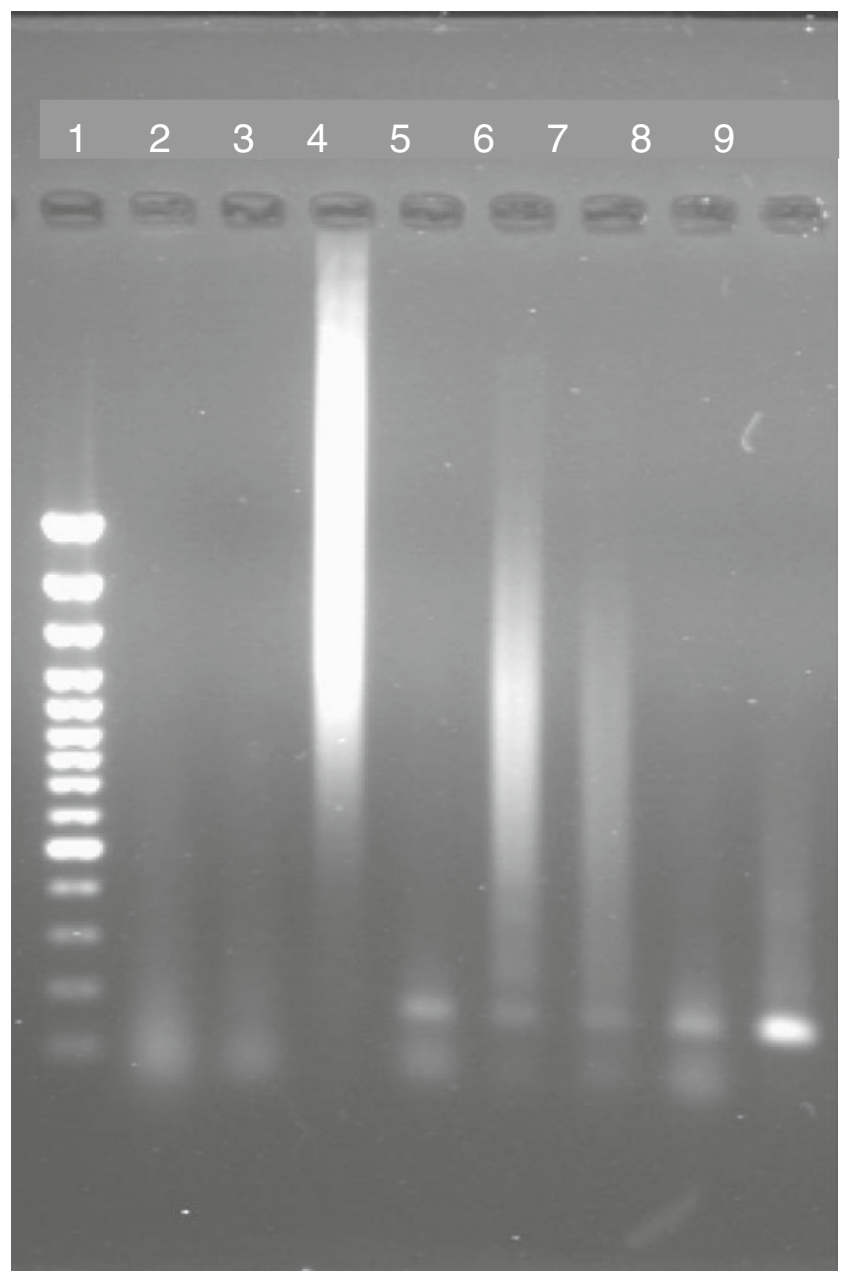

Fig. 1 1.5\% agarose gel electrophoresis of PCR products from buffy coat DNA of dogs. Lane 1 Standard marker, lanes 2 and 3 negative samples, lane 4 negative control, lane 5 standard Leishmania infantum (145 bp), lanes 6, 7, 8, and 9 positive dogs

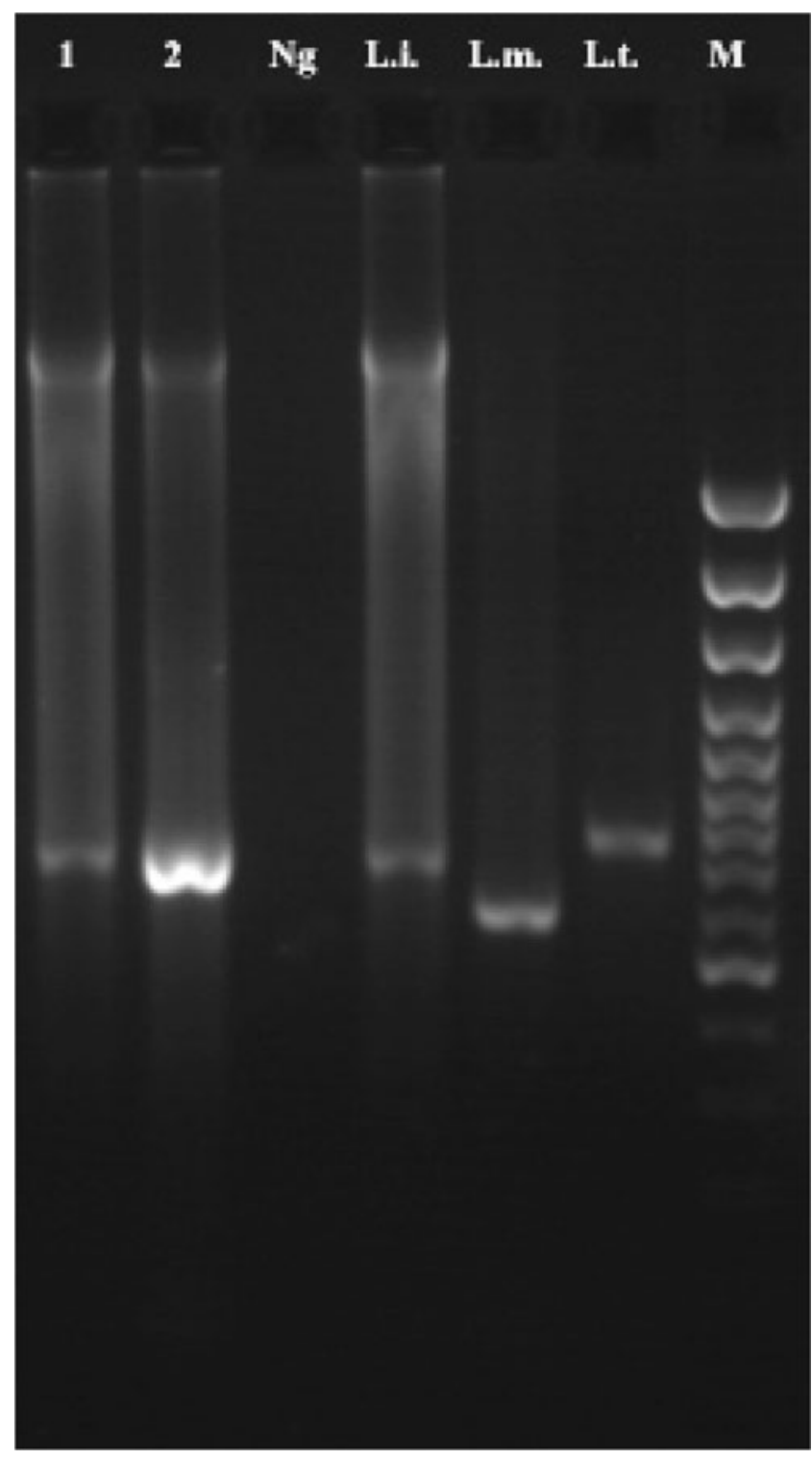

Fig. 2 1.5\% agarose gel electrophoresis of PCR products from buffy coat DNA of dogs. Lanes 1 and 2 DNA amplification of cases, lane 3 negative control, lane 4 standard Leishmania infantum (720 bp), lane 5 standard L. major (680 bp), lane 6 standard Leishmania tropica (760 bp), and lane 7 marker 
method the rate of infection in $<1$-year-old dogs $(46.1 \%)$ was higher than other age groups, whereas no significant differences between age groups were observed by serology. The high frequency of PCR positives in the young dogs might be due to greater susceptibility to infection or may to vertical transmission.

In our study, $88 \%$ (22/25) of PCR-positive and 67\% (4/ 6) seropositive subjects were asymptomatic. Since the majority of PCR-positive dogs were asymptomatic, indicating the presence of $L$. infantum amastigotes in the peripheral blood of the subjects, it clear that these asymptomatic dogs $(22 / 110,20 \%)$ are comparatively common in the study areas and may act as reservoir hosts in the transmission of L. infantum, to humans and to other dogs, by sandflies.

Moreira et al. (2007) showed that $5.4 \%$ of dogs without clinical symptoms can act as a reservoir. Moreover, SolanoGallego et al. (2001) reported more than 50\% (15 out of 26) of seropositive dogs were asymptomatic on clinical examination. Surprisingly, Guarga et al. (2000) showed that healthy skin of infected dogs is more attractive to sandflies. As the disease progress and skin status declines, the attraction of the female sandfly to the skin decreases. Consequently, this behavior of female sandflies can give good reason for the increase of the risk of infection in asymptomatic dogs in CVL endemic areas.

In a recent study of the role of asymptomatic dogs infected with $L$. infantum in an endemic focus of Iran by Moshfe et al. (2009), the concordance between DAT and PCR in symptomatic and asymptomatic dogs was $53.8 \%$ and $17 \%$, respectively. Consequently, DAT is not suitable to detect all dogs with asymptomatic $L$. infantum infections, but it can help to confirm diagnosis of symptomatic infected dogs. DAT is a simple, valid, and cost-effective test that can be recommended for seroprevalence surveys of symptomatic CVL in endemic areas. Our results in this case are consistent with previous studies that were carried out in other endemic regions of Iran (Edrissian et al. 1996; Mohebali et al. 2005; Moshfe et al. 2009).

Dogs (C. familiaris) are considered to be the principal reservoir host for MVL (Mohebali et al. 2005; Mazloumi Gavgani et al. 2002). Baldi et al. (2004) reported a close relation between HVL and CVL. The results of a previous study in Iran (Mazloumi Gavgani et al. 2002) showed that seroprevalence of children was significantly higher in villages with larger dog populations. However, the rate of the anti-leishmanial antibody in dogs from the northwest areas (Mazloumi Gavgani et al. 2002), central part (Fakhar et al. 2004), and different parts of Iran (Mohebali et al. 2005 ) was reported $43.3 \%, 22.8 \%, 25 \%$, and $14.2 \%$, respectively. In an earlier study (Edrissian et al. 1993), the rate of infection among dogs from endemic districts of Fars Province was $41.6 \%$. As shown, the prevalence is markedly below the values detected in dogs from other parts. It is also below that of other countries such as Greece (Sideris et al. 1999) and Spain (Fisa et al. 1999) which were 37\% and $10.2 \%$, respectively.

Moreover, Oliva et al. (2006) showed that the sensitivity of serologic and parasitological techniques is lower than nested PCR although it increased over time. Our results also showed PCR on BC from blood to be more sensitive than DAT.

Lachaud et al. (2001) compared advantages of whole blood (WB) with BC samples in PCR method. The use of WB is easier than that of $\mathrm{BC}$, particularly in field studies, but the latter yield a greater sensitivity because Leishmania parasites are obligate intracellular parasite of peripheral blood mononuclear cells and are therefore more concentrated in the BC. They also described that the methods using kDNA minicircle primers, such as RV1 and RV2, has been shown to be both highly sensitive and specific; it has detected the parasite in $100 \%$ of symptomatic and asymptomatic seropositive dogs with sensitivity equal to 0.0001 parasite $\mathrm{ml}^{-1}$ blood. As a whole, PCR is more sensitive in detecting the early stages of established infection and transient and self-limiting infections, whereas a reliable serological technique is more efficient in detecting advanced stages of infection, both in symptomatic and asymptomatic dogs, although some positive cases may convert to being seronegative during the course of infection (Gradoni 2002).

In conclusion, asymptomatic dogs as well as symptomatic ones, have an important role in the maintenance of $L$. infantum infection and most likely the concern of domestic cycle of the CVL in the MVL endemic areas. Moreover, PCR using BC samples is a valuable tool to detect the actual rate of infection in asymptomatic dogs that often have low or undetectable levels of IgG antibodies to Leishmania in endemic areas. In comparison, the results of the DAT revealed far fewer positives, if the results of the PCR are assumed to have indicated all the current infections. The main finding of our study demonstrated high prevalence of infection in nomadic dogs (27\%). These animals could contribute to the distribution of infection among other dogs and represent a potential risk for human health as well. Nomadic tribes move from South to North in spring and from North to South in autumn. They pass through cities and villages and have thus played an important role in the wide geographical distribution of VL in this province. It was shown that infected dogs play an important role in spreading disease in nonendemic foci of Italy with suitable conditions (Baldi et al. 2004).

There were no facilities to follow up the dogs so we cannot compare our result with other studies. Given the above observations, control of the dog reservoir and 
sandflies vectors, education of environmental health personnel, and well-established methods of diagnosis are required to prevent human infection. We suggest that for prevention of VL, nomadic tribes must be settled in village homes instead of tents where they have more exposure to environmental risk factors and fewer facilities. The surveillance of the disease in dogs and humans population can be monitored by clinical and serological approaches in health centers of the districts.

Acknowledgments The authors would like to thank the Office of Vice Chancellor for Research of Shiraz University of Medical Sciences, Shiraz University for financial support of this project (no. 84-2639) and the organization of nomadic affairs and health centers of Fars Province for providing the facility for the field sampling. The authors are grateful to Dr. M. Mohebali for DA antigen preparation, to K. Azizi and Dr. D. Mehrabani for helping in sample collection, and to Dr. H. Noyes for valuable comments and reading the manuscript.

\section{References}

Asgari Q, Fakhar M, Motazedian MH (2006) Nomadic kala-azar in south of Iran. Iran J Publ Health 35(3):85-86

Ashford DA, David JR, Freire M, David R, Sherlock I, Eulalio MC, Sampanio DP, Badaro R (1998) Studies on control of visceral leishmaniasis impact of dog control on canine and human visceral leishmaniasis in Jacobina, Bahia, Brazil. Am J Trop Med Hyg 59:53-57

Azizi K, Rassi Y, Javadian E, Motazedian MH, Rafizadeh S, Yaghoobi Ershadi MR, Mohebali M (2006) Phlebotomus (Paraphlebotomus) alexandri: a probable vector of Leishmania infantum in Iran. Ann Trop Med Parasitol 100:63-68

Baldi L, Mizzoni V, Guarino A (2004) Canine leishmaniasis in Campania: new and old foci (in Italian). Parasitologia 46:217-220

Edrissian GH, Ahanchin AR, Gharachahi AM, Chorbani M (1993) Serological studies of visceral leishmaniasis and search for animal reservoirs in Fars Province, southern Iran. Iran J Med Sci 18(3):99-105

Edrissian GH, Hajjaran H, Mohebali M, Soleimanzadeh G, Bokaei S (1996) Application and evaluation of direct agglutination test in sero-diagnosis of visceral leishmaniasis in man and canine reservoirs in Iran. Iran J Med Sci 21:119-124

Evans D (1989) Handbook of isolation, characterization and cryopreservation of Leishmania. UNDP/World Bank/WHO, $17-18$

Fakhar M, Mohebali M, Barani M (2004) Identification of endemic focus of Kala-azar and seroepidemiologcal study of visceral Leishmania infection in human and canine in Qom Province. Armaghan-Danesh 9(3):43-52

Fakhar M, Motazedian MH, Asgari Q, Mohebali M, Mehrabani D (2006) A new endemic focus of visceral leishmaniosis in southern Iran. Armaghan-Danesh 11(2):104-110

Fakhar M, Motazedian MH, Hatam GR, Asgar Q, Kalantari M, Mohebali M (2008) Asymptomatic human carriers of Leishmania infantum: possible reservoirs for Mediterranean visceral leishmaniasis in southern Iran. Ann Trop Med Parasitol 102(7):577583

Fars Budget and Planning Organization (2003) Fars census (2003). Management and Planning Press, Tehran

Fisa R, Gàllego M, Castillejo S, Aisa MJ, Serra T, Riera C, Carri J, Gàllego J, Portus M (1999) Epidemiology of canine leishmaniasis in
Catalonia (Spain). The example of the Priorat focus. Vet Parasitol 83:87-97

Gradoni L (2002) The dignosis of canine leishmaniasis. In: Canine leishmaniasis: moving towards a solution. Proceedings of the second international canine leishmaniasis forum. Sevilla, Spain

Guarga J, Moreno L, Lucientes JJ, Gracia MJ, Penibanez MAJ, Alvar J, Castillo JA (2000) Canine leishmaniasis transmission: higher infectivity amongst naturally infected dogs to sand flies is associated with lower proportions of T helper cells. Acta Trop 77:203-207

Harith A, Salappendel RJ, Reiter I, Knapen F, Korte P, Huigen E, Kolk RHG (1989) Application of a direct agglutination test for detection of specific anti-Leishmania antibodies in the canine reservoir. J Clin Microbiol 27:2252-2257

Koutinas AF, Polizopoulou ZS, Saridomichelakis MN, Argyriadis D, Fytianou A, Plevraki KG (1999) Clinical considerations on canine visceral leishmaniasis in Greece: a retrospective study of 158 cases (1989-1996). J Am Anim Hosp Assoc 35:376-383

Lachaud L, Chabbert E, Dubessay P, Reynes J, Lamothe J, Bastien P (2001) Comparison of various sample preparation methods for PCR diagnosis of visceral leishmaniasis using peripheral blood. J Clin Microbiol 39(2):613-617

Leontides LS, Saridomichelakis MN, Billinis C, Kontos V, Koutinas AF, Galatos AD, Mylonakis ME (2002) A cross-sectional study of Leishmania spp. infection in clinically healthy dogs with polymerase chain reaction and serology in Greece. Vet Parasitol 109(1-2):19-27

Maia C, Ramada J, Cristovao JM, Goncalves L, Campino L (2009) Diagnosis of canine leishmaniasis; conventional and molecular techniques using different tissues. Vet J 179(1):142-144

Mazloumi Gavgani AS, Mohite H, Edrissian GH, Mohebali M, Davies CR (2002) Domestic dog ownership in Iran is a risk factor for human infection with Leishmania infantum. Am J Trop Med Hyg 67(5):511-515

Mohebali M, Hamzavi Y, Edrissian GH, Forouzani A (2001) Seroepidemiological study of visceral leishmaniasis among humans and animal reservoirs in Bushehr Province, Islamic Republic of Iran. East Mediterr Health J 7(6):912-917

Mohebali M, Hajjaran H, Hamzavi Y, Mobedi I, Arshi S, Zarei Z, Akhoundi B, Naeini K, Manouchehri AR, Fakhar M (2005) Epidemiological aspects of canine visceral leishmaniosis in the Islamic Republic of Iran. Vet Parasitol 129(3):243-252

Molina R, Lohse JM, Pulido F, Laguna F, Lopez-Velez R, Alva J (1999) Infection of sand flies by humans infected with Leishmania infantum and human immunodeficiency virus. Am J Trop Med Hyg 60:51-53

Moreira MA, Luvizotto MC, Garcia JF, Corbett CE, Laurenti MD (2007) Comparison of parasitological immunological and molecular methods for the diagnosis of leishmaniasis in dogs with different clinical signs. Vet Parasitol 145(3-4):245-252

Moshfe A, Mohebali M, Edrissian GH, Zarei Z, Akhoundi B, Kazemi B, Jamshidie S, Mahmoodi M (2008) Seroepidemiological study on canine visceral leishmaniasis in Meshkin-Shahr District, Ardabil Province, northwest of Iran during 2006-2007. Iran J Parasitol 3(3):1-10

Moshfe A, Mohebali M, Edrissian GH, Zarei Z, Akhoundi B, Kazemi B, Jamshidie S, Mahmoodi M (2009) Canine visceral leishmaniasis: asymptomatic infected dogs as a source of $L$. infantum infection. Acta Trop 112:101-105

Motazedian H, Karamian M, Noyes HA, Ardehali S (2002) DNA extraction and amplification of Leishmania from archived, Giemsa-stained slides, for the diagnosis of cutaneous leishmaniasis by PCR. Ann Trop Med Parasitol 96:31-34

Oliva G, Scalone A, Foglia Manzillo V, Gramiccia M, Pagano A, Di Muccio T, Gradoni L (2006) Incidence and time course of Leishmania infantum infections examined by parasitological, 
serologic and nested-PCR techniques in a cohort of naive dogs exposed to three consecutive transmission seasons. J Clin Microbiol 44:1318-1322

Sideris V, Papadopoulou G, Dotsika E, Karagouni E (1999) Asymptomatic canine leishmaniasis in Greater Athens area, Greece. Eur J Epidemiol 15:271-276
Solano-Gallego L, Morell P, Arboix M, Alberola J, Ferrer L (2001) Prevalence of Leishmania infantum infection in dogs living in an area of canine leishmaniasis endemicity using PCR on several tissues and serology. J Clin Microbiol 39:560-563

World Health Organization (1998) Life in twenty first century: a vision for all. World Health Report (WHO), Geneva, Switzerland 